\title{
Hierarchical Regulation of Odorant Receptor Gene Choice and Subsequent Axonal Projection of Olfactory Sensory Neurons in Zebrafish
}

\author{
Yuki Sato, ${ }^{1}$ Nobuhiko Miyasaka, ${ }^{1,2}$ and Yoshihiro Yoshihara ${ }^{1,2}$ \\ ${ }^{1}$ Laboratory for Neurobiology of Synapse, RIKEN Brain Science Institute, Wako, Saitama 351-0198, Japan, and ${ }^{2}$ Core Research for Evolutional Science and \\ Technology, Japan Science and Technology Agency, Osaka 560-0082, Japan
}

\begin{abstract}
In both Drosophila and mice, olfactory sensory neurons (OSNs) expressing a given odorant receptor (OR) project axons to specific glomeruli in the antennal lobe or olfactory bulb $(\mathrm{OB})$, developing a topographic odor map. To gain insights into the modes of OR expression and axonal projection in zebrafish, we generated a bacterial artificial chromosome transgenic line carrying an OR gene cluster in which two OR-coding sequences, OR111-7 and OR103-1, were replaced with yellow fluorescent protein (YFP) and cyan fluorescent protein (CFP), respectively. In the transgenic embryos, YFP and CFP signals appear in small populations of OSNs at an early stage of development when OR expression is first observed. Time-lapse imaging of living embryos revealed that both YFP- and CFP-expressing OSNs project axons to the medial portion of the $\mathrm{OB}$. This pattern of axonal projection is maintained in the adult transgenic fish, in which fluorescently labeled OSN axons target a topographically fixed cluster of glomeruli in the medial OB. Because the OR-coding sequences were replaced with fluorescent reporter genes, we examined which OR genes are expressed in YFP/CFP-expressing OSNs and found that the OR choice is mostly restricted to OR members within the same subfamily of the cluster. Furthermore, we found that the one receptor- one neuron rule is not always applicable to zebrafish OSNs and that multiple receptors- one neuron is true for a subpopulation of OSNs in both wild-type and transgenic fish. These data demonstrate two distinct modes of OR expression and suggest a model of the hierarchical regulation of OR gene choice and subsequent axonal projection in the zebrafish olfactory system.
\end{abstract}

Key words: olfactory bulb; glomerulus; odor map; axon guidance; teleost olfactory system; fluorescent protein

\section{Introduction}

Individual olfactory sensory neurons (OSNs) in mice express only one conventional odorant receptor $(\mathrm{OR})$ gene of a repertoire of 1300 possibilities (Chess et al., 1994; Malnic et al., 1999), which defines the functional identity of each OSN. OSNs expressing a given $\mathrm{OR}$ converge their axons onto a few defined glomeruli within the olfactory bulb (OB) (Ressler et al., 1994; Vassar et al., 1994; Mombaerts et al., 1996), providing a topographic odor map in the brain. In addition, the OR protein is responsible for axonal guidance of OSNs and the selective expression of OR genes (Wang et al., 1998; Serizawa et al., 2003; Feinstein and Mombaerts, 2004; Lewcock and Reed, 2004; Shykind et al., 2004). Thus, the choice of one functional OR in an OSN and its axonal projection to a specific glomerulus are fundamental bases for the organization and functions of the olfactory system.

Received Sept. 26, 2006; revised Dec. 18, 2006; accepted Jan. 8, 2007.

This work was supported in part by a Grant-in-Aid for Scientific Research (B) (Y.Y.) and a Grant-in-Aid for Young Scientists (N.M.) from the Ministry of Education, Culture, Sports, Science, and Technology of Japan. We thank N. Tamamaki (Kumamoto University, Kumamoto, Japan) for the gift of the rabbit anti-GFP antibody; M. Lalioti (University of Birmingham, Birmingham, UK) for the gift of pKOV-KanF and pDF25-Tet; N. Heintz (Rockefeller University, New York, NY) for the gift of pSV1.RecA; S. Yamashita, C. Ito, and K. Sanada for maintenance of our zebrafish facility; and all members of Yoshihara laboratory for helpful discussions.

Correspondence should be addressed to Dr. Yoshihiro Yoshihara, Laboratory for Neurobiology of Synapse, RIKEN Brain Science Institute, 2-1 Hirosawa, Wako, Saitama 351-0198, Japan. E-mail: yoshihara@brain.riken.jp. D01:10.1523/JNEUROSCI.4218-06.2007

Copyright $\odot 2007$ Society for Neuroscience $\quad$ 0270-6474/07/271606-10\$15.00/0
Zebrafish provide an attractive vertebrate model system in which to investigate molecular and cellular mechanisms underlying the development and functions of the olfactory system. The zebrafish olfactory system contains molecular constituents, cell types, and overall organization that are all very similar to those of mice. Functional imaging of $\mathrm{OB}$ glomeruli in zebrafish demonstrated that different odorants elicit defined patterns of glomerular activity (Friedrich and Korsching, 1997, 1998), indicating the existence of a topographic odor map also in fish. Besides these organizational and functional similarities, the numbers of OR genes and $\mathrm{OB}$ glomeruli in zebrafish are smaller than those in mice by an order of magnitude (Baier and Korsching, 1994; Friedrich and Korsching, 1997; Alioto and Ngai, 2005; Niimura and Nei, 2005), enabling us to reduce the complexity of questions on OR choice and glomerular targeting mechanisms. The fish olfactory epithelium (OE) contains two major types of OSNs, ciliated and microvillous neurons. These two types of OSNs express distinct types of chemosensory receptors, project their axons to different regions of the $\mathrm{OB}$, and probably mediate different types of behaviors (Cao et al., 1998; Morita and Finger, 1998; Speca et al., 1999; Hamdani et al., 2001; Hamdani and Døving, 2002; Hansen et al., 2003, 2004; Weltzien et al., 2003; Sato et al., 2005). In situ hybridization analyses revealed that individual ORs are typically expressed in a small population of OSNs (Ngai et al., 1993; Barth et al., 1997; Speca et al., 1999; Sato et al., 2005). 
However, the modes of OR choice and axonal projection of OSNs expressing a given $\mathrm{OR}$ are not well understood in fish.

Here, we generated a bacterial artificial chromosome (BAC) transgenic zebrafish line carrying an OR gene cluster in which two OR-coding sequences, OR111-7 and OR103-1, were replaced with yellow fluorescent protein (YFP) and cyan fluorescent protein (CFP), respectively. In the transgenic fish, fluorescently labeled OSNs expressed limited members of OR genes and projected their axons to spatially invariant glomeruli within the OB. These observations suggest a model of the hierarchical regulation of OR gene choice and subsequent axonal projection in the zebrafish olfactory system.

\section{Materials and Methods}

Animals. Zebrafish, Danio rerio, were maintained in our laboratory as described previously (Westerfield, 1995). Embryos were incubated at $28.5^{\circ} \mathrm{C}$ and staged according to hours postfertilization and morphological criteria (Kimmel et al., 1995).

BAC transgene construction. The BAC Zebra Fish Library (Genome Systems, St. Louis, MO) was screened by PCR to obtain a clone containing the zebrafish OR gene cluster previously characterized (Barth et al., 1997; Dugas and Ngai, 2001; Alioto and Ngai, 2005). The 100G14 BAC clone was used to construct a BAC-YC transgene by two steps of targeted BAC modifications as follows.

The first BAC modification was the replacement of the OR111-7 coding sequence with gap-YFP cDNA. A couple of homology arms containing $0.8 \mathrm{~kb}$ fragments of $5^{\prime}$ and $3^{\prime}$ flanking regions of the OR111-7 coding sequence were amplified from the BAC DNA by PCR and subcloned. The gap-YFP cDNA (pEYFP-Mem; BD Biosciences Clontech, Palo Alto, CA) was flanked by the homology arms and inserted into the SalI site of pKOV-KanF vector (Lalioti and Heath, 2001) to create a shuttle vector. Using the shuttle vector and the pDF25-Tet plasmid, homologous recombination of BAC DNA was performed as described previously (Lalioti and Heath, 2001) to generate a modified BAC clone BAC[OR111-7:gap-YFP].

The second modification was the replacement of the OR103-1 coding sequence with gap-CFP cDNA. For a shuttle vector, the gap-CFP cDNA (pECFP-Mem; BD Biosciences Clontech) was flanked by a couple of 0.5 $\mathrm{kb}$ homology arms for the OR103-1 coding sequence and inserted into the SalI site of pSV1.RecA vector (Yang et al., 1997). Targeted BAC modification was performed with the clone BAC[OR111-7:gap-YFP] as described previously (Yang et al., 1997) to obtain a construct BAC[OR1117:gap-YFP/OR103-1:gap-CFP] (abbreviated as BAC-YC construct), in which the coding sequences of OR111-7 and OR103-1 were replaced with gap-YFP and gap-CFP, respectively.

All of the PCR fragments used in the construction were subjected to DNA sequencing, and several modified BAC clones isolated in individual steps of the modification were screened by Southern blot analysis.

Generation of transgenic zebrafish. The modified BAC transgene was purified using QIAfilter Plasmid Maxi kit (Qiagen, Hilden, Germany) and diluted to $120 \mathrm{ng} / \mu \mathrm{l}$ in distilled water containing $0.1 \%$ phenol red. The DNA solution was injected into cytoplasm of one-cell stage embryos, and the injected embryos with fluorescence in OSNs were raised to sexual maturity. Germ line-transmitting founders were screened by PCR with genomic DNA extracted from their progeny using a pair of primers for the green fluorescent protein (GFP) gene. One line of transgenic fish was generated and termed Tg (BAC[OR111-7:gap-YFP/OR103-1:gap-CFP] $)^{\text {rw039 }}$ (abbreviated as BAC-YC line).

The transgenic zebrafish lines $\mathrm{Tg}\left(\mathrm{OMP}^{2 \mathrm{k}}\right.$ :gap-YFP) ${ }^{\text {rw032a }}$ (OMP: YFP) and $\mathrm{Tg}\left(\mathrm{OMP}^{2 \mathrm{k}} \text { :lyn-mRFP }\right)^{\text {rw035a }}$ (OMP:RFP) were described previously (Miyasaka et al., 2005; Sato et al., 2005). All of the transgenic zebrafish lines including Tg (BAC[OR111-7:gap-YFP/OR103-1:gap$C F P])^{\text {rw039a }}$ can be obtained from us under the support of Zebrafish National BioResource Project of Japan (www.shigen.nig.ac.jp/ zebra/index_en.html).

Time-lapse imaging of OSN projection in zebrafish embryos. Time-lapse imaging of OSN projection in zebrafish embryos was performed as described previously (Sato et al., 2005).
Immunohistochemistry. Seven- to 13-month-old adult fish $(\sim 3.5 \mathrm{~cm}$ body length) were anesthetized with $0.016 \%$ ethyl- $m$-aminobenzoate methanesulphonate (tricaine) (Nacalai Tesque, Kyoto, Japan), and tissues were dissected out.

For immunostaining of OE sections, cryostat slices were prepared as described previously (Sato et al., 2005). OE sections (14 $\mu \mathrm{m}$ thickness) from adult BAC-YC fish were incubated with rat anti-GFP monoclonal antibody (GF090R, rat IgG2a, 1:1000; Nacalai Tesque) and then incubated with biotin-conjugated goat anti-rat IgG (1:500; Jackson ImmunoResearch, West Grove, PA), followed by staining with a Vectastain ABC elite kit (Vector Laboratories, Burlingame, CA). Signals were visualized with the $\mathrm{Ni}^{2+}$-intensified diaminobenzidine (DAB)/peroxide reaction. OE sections (10 $\mu \mathrm{m}$ thickness) from adult BAC-YC;OMP:RFP double-transgenic fish were incubated with rat anti-GFP monoclonal antibody (1:1000; Nacalai Tesque) and subsequently incubated with AlexaFluor488-conjugated goat anti-rat IgG (1:300; Invitrogen, Carlsbad, CA). Fluorescent signals of AlexaFluor488 and red fluorescent protein (RFP) were imaged by a confocal laser scanning microscope (FV500; Olympus Optical, Tokyo, Japan).

Whole-mount immunohistochemistry was performed essentially as described previously (Macdonald, 1999; Miyasaka et al., 2005) with some modifications. Briefly, telencephalic hemispheres together with olfactory epithelia were fixed in $4 \%$ paraformaldehyde (PFA) in PBS overnight at $4^{\circ} \mathrm{C}$. After permeabilization with acetone, tissues were incubated with $0.3 \% \mathrm{H}_{2} \mathrm{O}_{2}$ to quench endogenous peroxidase activities, blocked with $10 \%$ normal goat serum in PBS containing $1 \%$ dimethylsulfoxide and $0.1 \%$ Tween-20 (PBDT), and incubated overnight at $4^{\circ} \mathrm{C}$ with rabbit polyclonal anti-GFP antibody (1:500; a kind gift from Dr. N. Tamamaki, Kumamoto University, Kumamoto, Japan) diluted in PBDT. The tissues were then incubated with biotin-conjugated goat anti-rabbit IgG (1:500; Jackson ImmunoResearch), followed by staining with a Vectastain ABC elite kit. Signals were visualized with the $\mathrm{DAB} /$ peroxide reaction. The tissues were postfixed with $4 \%$ PFA overnight at $4^{\circ} \mathrm{C}$, washed with PBS, and examined with a light microscope (MVX10; Olympus Optical). Images at $10 \mu \mathrm{m}$ steps were acquired and stacked for composition of infocus images using Dynamic Eye software (Mitani, Fukui, Japan).

Immunostaining of $\mathrm{OB}$ sections from adult BAC-YC;OMP:RFP double-transgenic fish was performed as described previously (Sato et al., 2005).

Analysis of OR gene expression. DNA fragments of zebrafish OR genes were amplified by PCR from the zebrafish genomic DNA and the 100G14 BAC DNA using specific primers for each OR gene. PCR primers were designed on the basis of predicted coding sequences for zebrafish OR genes (Niimura and Nei, 2005) and the DNA sequence of the 100G14 BAC clone (supplemental Table S1, available at www.jneurosci.org as supplemental material). PCR products containing full or partial length of OR-coding sequences were subcloned into pGEM-T Easy vector (Promega, Madison, WI) and used as templates for cRNA probes. In situ hybridization was performed essentially as described previously (Inaki et al., 2004; Sato et al., 2005).

For analysis of coexpression of the GFP variants with OR genes in adult BAC-YC fish, OE sections (10-14 $\mu \mathrm{m}$ thickness) were subjected to fluorescent in situ hybridization using 2-hydroxy-3-naphtoic acid-2phenylanilide phosphate (HNPP)/Fast Red (Roche, Mannheim, Germany) and subsequently immunostained with rabbit anti-GFP polyclonal antibody (1:1000; BD Biosciences Clontech). At least 50 GFPimmunoreactive OSNs derived from several individuals were examined for each OR probe, and the percentages of the OR-positive cells among GFP-immunoreactive cells were calculated.

For double-color in situ hybridization, cRNA probes were labeled with digoxigenin or fluorescein and used for hybridization to OE sections (10 $\mu \mathrm{m}$ thickness). After hybridization, sections were washed, treated with blocking reagent (Roche), and incubated overnight at room temperature (RT) with a mixture of alkaline phosphatase-conjugated antidigoxigenin antibody (1:1000; Roche) and horseradish peroxidaseconjugated anti-fluorescein antibody (1:100; Roche). After washing in $100 \mathrm{~mm}$ Tris- $\mathrm{HCl}, \mathrm{pH} 7.5$, containing $150 \mathrm{~mm} \mathrm{NaCl}$ and $0.05 \%$ Tween 20 (TNT), the sections were incubated with biotinyl tyramide (1:50, TSA Biotin System; PerkinElmer, Wellesley, MA) at RT for $10 \mathrm{~min}$, washed in 
TNT, and incubated with streptavidinconjugated AlexaFluor488 (1:300; Invitrogen) at RT for $30 \mathrm{~min}$. For detection of alkaline phosphatase, the sections were incubated with HNPP/Fast Red (Roche).

To estimate the numbers of cells hybridizing to individual OR probes in an entire olfactory rosette, OE sections (10 $\mu \mathrm{m}$ thickness) from adult wild-type fish were examined by either single or double in situ hybridization with digoxigenin- and/or fluorescein-labeled cRNA probes. Detection was accomplished via the use of alkaline phosphataseconjugated anti-digoxigenin antibody (1:1000; Roche) followed by HNPP/Fast Red (Roche) or the use of peroxidase-conjugated anti-digoxigenin (1:100; Roche) or antifluorescein (1:100; Roche) antibody followed by biotinyl tyramide (PerkinElmer) and streptavidin-conjugated AlexaFluor488 (1:300; Invitrogen). For each OR probe, every four slices from serial OE sections ( $10 \mu \mathrm{m}$ thickness) were hybridized, and the total number of OR probe-positive cells was counted. Different individual rosettes $(n=3-7)$ were examined for each OR probe.

Nomenclature and genomic distribution of zebrafish OR genes. The organization of an OR gene cluster on chromosome 15 was based on the array of OR genes that was described previously (Dugas and Ngai, 2001). The new nomenclature for zebrafish ORs (Alioto and Ngai, 2005) was adopted in this manuscript. The OR nomenclature equivalents were provided in supplemental Table S1 (available at www.jneurosci.org as supplemental material). Chromosomal localization of individual ORs followed the data from Niimura and Nei (2005).

\section{Results}

\section{Construction of a BAC OR transgene tagged with two} fluorescent reporters

Among 150 members of the zebrafish OR gene family, a total of 22 functional OR genes belonging to five subfamilies (OR102, OR103, OR111, OR107, and OR119) are clustered within a $140 \mathrm{~kb}$ region on the zebrafish chromosome 15 (Dugas and Ngai, 2001; Alioto and Ngai, 2005) (Fig. 1A). We screened a zebrafish BAC library and isolated a clone containing an array of 16 functional OR genes belonging to OR102, OR103, and OR111 subfamilies within a $95 \mathrm{~kb}$ stretch of genomic DNA (Fig. $1 B$ ). To visualize functional subsets of OSNs with distinct fluorescent proteins, the BAC clone was modified by replacement of the coding sequences of OR111-7 and OR103-1 with membrane-targeted fluorescent reporters, gap-YFP and gap-CFP, respectively (Fig. 1C), and used to generate a transgenic zebrafish line BAC-YC (OR111-7:YFP/ OR103-1:CFP). OR111-7 and OR103-1 were chosen for replacement because of early onsets of expression during development and relatively large numbers of OSNs expressing these two ORs (Barth et al., 1996, 1997). We have shown previously that OR111-7 and OR103-1 are expressed in a subset of ciliated OSNs (Sato et al., 2005).

\section{BAC transgene expression in small populations of OSNs during development}

Zebrafish is an excellent model organism for studies in development neurobiology because of its external fertilization, rapid development, and transparency of embryos. These advantageous properties in combination with transgenic expression of fluorescent proteins enable us to perform in vivo time-lapse observation of axon growth dynamics in living zebrafish embryos. We established a transgenic zebrafish line, OMP:RFP, in which membrane-targeted RFP is expressed in almost all ciliated OSNs under the control of zebrafish olfactory marker protein (OMP) promoter (Sato et al., 2005). To examine the expression patterns of YFP and CFP in the BAC-YC transgenic fish, we obtained double-transgenic fish by crossing the BAC-YC line with the OMP:RFP line. In BAC-YC;OMP:RFP double-transgenic embryos, fluorescent signals of YFP and CFP were detected by $1.5 \mathrm{~d}$ postfertilization ( $\mathrm{dpf}$ ) when several endogenous OR genes begin to be expressed (Barth et al., 1997) (Fig. 2A,D,G). At this stage, YFP and CFP were expressed in small numbers of OSNs, and these two populations appeared to be primarily overlapping. Outgrowing axons of YFP- and CFP-labeled OSNs extended toward the forebrain along the preceding RFP-labeled axons (Fig. $2 A, D, G)$. YFP- and CFP-labeled OSNs greatly increased in number by 2-3 dpf, and the two differentially labeled OSN populations gradually segregated in the OE (Fig. $2 B, E, H$ ). In the presumptive OB, RFP-labeled axons projected to form many protoglomerular structures, whereas YFP- and CFP-labeled axons shaped a specific cluster of protoglomeruli in the medial portion (Fig. $2 B, E, H$ ). Such topographically fixed axonal targeting of YFP- and CFP-labeled OSNs to the medial glomeruli in the $\mathrm{OB}$ was clearly observed at $5 \mathrm{dpf}$ when glomerular structures become evident (Fig. 2C,F,I). Although YFP- and CFP-labeled OSN populations were distinct in the OE, their axons targeted the same cluster of glomeruli (Fig. $2 H, I$ ).

\section{Selective innervation of BAC transgene-expressing OSN axons to a medial glomerular cluster in the adult $\mathrm{OB}$}

In BAC-YC embryos, YFP- and CFP-labeled OSNs projected their axons to a specific cluster of protoglomeruli in the medial region of the $\mathrm{OB}$ (Fig. 2). We next examined whether this pattern of axonal projection persists in adult BAC-YC fish. Fluorescence of neither YFP nor CFP could be detected directly in the $\mathrm{OE}$ and $\mathrm{OB}$ from adult BAC-YC fish. For this reason, we adopted whole-mount $\mathrm{OE} / \mathrm{OB}$ immunostaining with the anti-GFP antibody detecting both YFP and CFP, although we could not discriminate two fluorescent proteins. In the OMP:YFP line as a control, YFP-expressing ciliated OSNs projected their axons to a large number of glomeruli distributed widely in the dorsal and medial regions of the $\mathrm{OB}$ (Fig. $3 A-C$ ). In contrast, only a small cluster of glomeruli located in the anteromedial portion of the $\mathrm{OB}$ was targeted by GFP-immunoreactive YFP/CFP-expressing OSNs in the BAC-YC line (Fig. 3D-F).

We also investigated a series of horizontal sections through the $\mathrm{OB}$ of adult BAC-YC;OMP:RFP double-transgenic fish. GFPimmunoreactive OSN axons approached and entered the OB from the ventral side (Fig. 4D,E, arrowheads) and converged onto a cluster of glomeruli in the medial portion of the $\mathrm{OB}$ at the intermediate level along the dorsoventral axis (Fig. 4B,C). Merged images of anti-GFP and anti-RFP labeling revealed that GFPimmunoreactive glomeruli were completely included in RFP- 


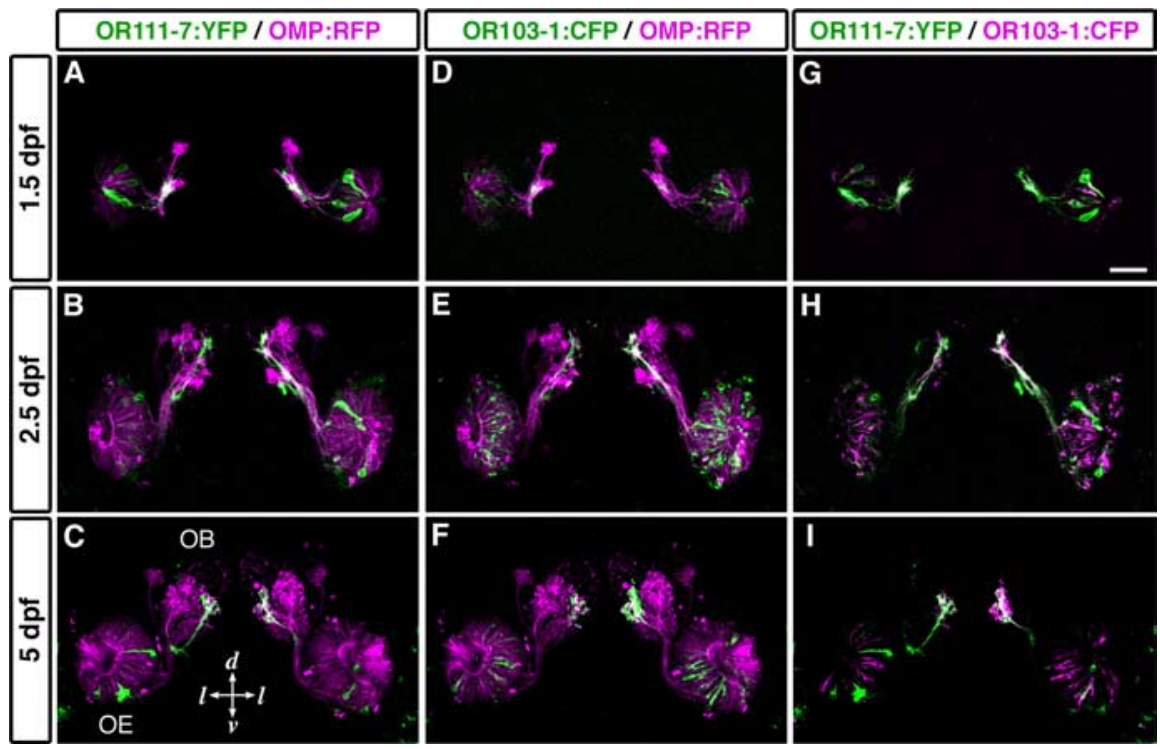

Figure 2. Expression of YFP and CFP in subpopulations of OSNs in a living embryo. Labeled OSNs in a representative BAC-YC OMP:RFP double-transgenic embryo were observed at $1.5 \mathrm{dpf}(\boldsymbol{A}, \boldsymbol{D}, \boldsymbol{G}), 2.5 \mathrm{dpf}(\boldsymbol{B}, \boldsymbol{E}, \boldsymbol{H})$, and $5 \mathrm{dpf}(\boldsymbol{C}, \boldsymbol{F}, \boldsymbol{I})$. YFP-labeled (green in $\boldsymbol{A}-\boldsymbol{C}, \boldsymbol{G}-\boldsymbol{I}$ ), (FP-labeled (green in $\boldsymbol{D}-\boldsymbol{F}$; magenta in $\mathbf{G} \boldsymbol{I}$ ), and RFP-labeled (magenta in $\boldsymbol{A}-\boldsymbol{F}$ ) OSNs are shown. All photographs represent stacked images of optical sections. YFP- and CFP-labeled OSNs project their axons to a specific cluster of glomeruli in the medial portion of the OB. d, Dorsal; v, ventral; I, lateral. Scale bar, $50 \mu \mathrm{m}$.
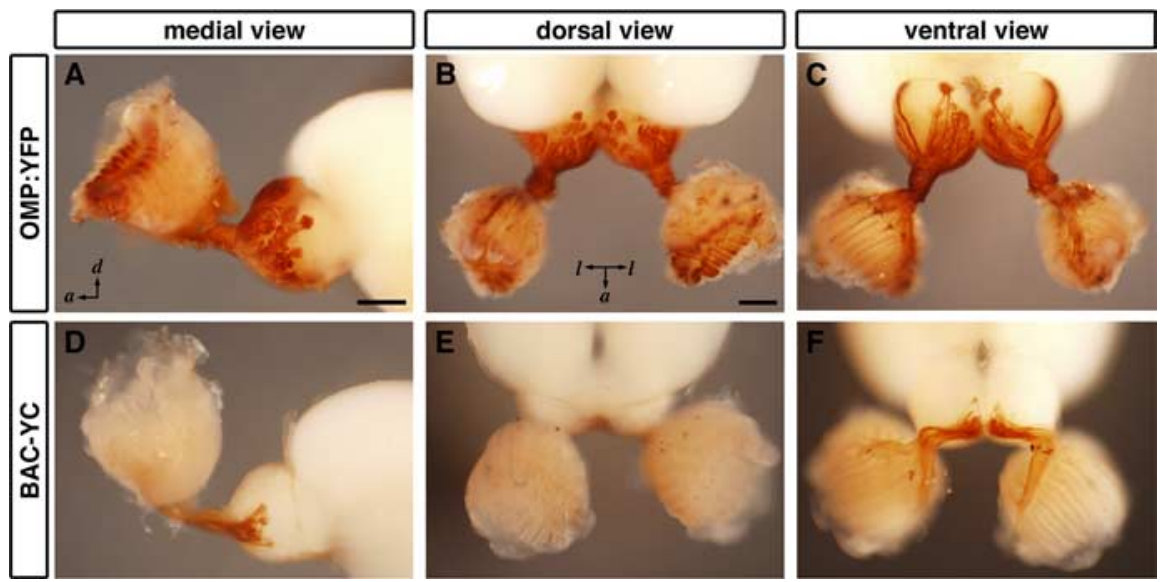

Figure 3. Projection of YFP/CFP-expressing OSN axons to the medial region of the OB in adult BAC-YC fish. Whole-mount OBs together with OE were stained with anti-GFP antibody. $A-C$, In adult OMP:YFP fish, YFP-labeled OSNs project their axons widely to a number of glomeruli in the dorsal and ventromedial OB. $\boldsymbol{D}-\boldsymbol{F}$, In adult BAC-YC fish, axonal projection of GFP-immunoreactive YFP/CFP-expressing OSNs is observed only in a small portion of the medial OB. $\boldsymbol{A}, \boldsymbol{D}$, Medial views; $\boldsymbol{B}, \boldsymbol{E}$, dorsal views; $\boldsymbol{C}, \boldsymbol{F}$, ventral views. a, Anterior; d, dorsal; I, lateral. Scale bars: (in $\boldsymbol{A}), \boldsymbol{A}, \boldsymbol{D}, 200 \mu \mathrm{m}$; (in $\boldsymbol{B}), \boldsymbol{B}, \boldsymbol{C}, \boldsymbol{E}, \boldsymbol{F}, 200 \mu \mathrm{m}$

labeled glomeruli (Fig. $4 F-J$ ), suggesting that YFP and CFP are expressed in a subset of RFP-expressing ciliated OSNs. Counterstaining with the antibody against a synaptic vesicle protein SV2 for definition of glomerular structures demonstrated that GFPimmunoreactive axons definitely innervated a small number of glomeruli (Fig. $4 L-N$ ). The patterns of axonal targeting from GFP-immunoreactive OSNs in the BAC-YC line were almost same between the right and left OBs and among different individuals tested (data not shown).

\section{Expression of YFP/CFP in a subpopulation of ciliated OSNs} in the adult $\mathrm{OE}$

The axonal trajectory of GFP-immunoreactive OSNs in BAC-YC fish was completely included in that of ciliated OSNs (Figs. 3, 4), suggesting that YFP and CFP are expressed in a subset of ciliated
OSNs. This idea is likely, because the replaced ORs, OR111-7 and OR103-1, are expressed exclusively in ciliated OSNs (Sato et al., 2005). Ciliated and microvillous OSNs in fish can be distinguished by their different morphologies and relative positions in the OE. The ciliated OSNs with long dendrites are situated in the deep layer of the OE, whereas microvillous OSNs with short dendrites are located in the superficial layer (Morita and Finger, 1998; Hansen et al., 2003, 2004). To confirm that YFP/CFP was actually expressed in ciliated OSNs, OE sections from adult BAC-YC fish were immunostained with the antibody against GFP. GFP immunoreactivity was observed in a subpopulation of cells that were distributed sparsely within the sensory area of the OE (Fig $5 A)$. Almost all labeled OSNs possessed long dendrites, and their somata were located in the deep layer of the OE (Fig. 5B), representing the features of ciliated OSNs. In addition, double-fluorescent labeling of OE sections from BAC-YC;OMP:RFP double-transgenic fish revealed that all of the GFP-immunoreactive cells were included in the population of RFP-labeled ciliated OSNs (Fig. 5C). These findings indicate that YFP and CFP inserted into the two OR gene loci within the BAC-YC transgene are allowed to be expressed exclusively in ciliated OSNs as well as endogenous ORs.

\section{Expression of OR genes belonging to} limited subfamilies in

\section{YFP/CFP-expressing OSNs}

Although we succeeded in visualization of a specific subset of OSNs by using BAC transgenesis, there still remains an important open question as to which OR genes are expressed in YFP/CFP-expressing OSNs. Because we failed to induce bicistronic expression of the OR-coding sequence and the fluorescent reporter gene using an internal ribosome entry site in zebrafish (data not shown), the coding sequences of OR111-7 and OR103-1 were replaced with fluorescent reporter genes in the BAC-YC construct (OR111-7:YFP/OR1031:CFP). Previous studies with transgenic mice showed that OSNs expressing transgenes with an OR pseudogene or a truncated OR-coding sequence transcribe an additional OR gene, whereas OSNs expressing a functional OR do not (Serizawa et al., 2003; Lewcock and Reed, 2004). It has been also reported that OSNs initially expressing a mutant OR switch to express alternate ORs at high frequencies (Shykind et al., 2004). These findings suggested that the expression of a functional OR elicits a feedback signal that terminates OR switching and abrogates expression of additional OR genes. If a similar regulation mechanism of OR gene expression exists in the zebrafish olfactory system, OSNs that made the first expression choice on the fluorescent reporters would make the second choice on additional OR genes. 
To determine which OR genes are expressed in YFP/CFP-expressing OSNs, OE sections from adult BAC-YC fish were hybridized with cRNA probes for OR genes and immunostained with the antibody against GFP. First, we investigated whether the YFP/CFP-expressing OSNs chose the replaced OR, OR111-7 or OR103-1, from the endogenous alleles. A careful examination of double-labeled OE sections clarified that only a small population of GFP-immunoreactive OSNs expressed OR111-7 (5.8\%) or OR103-1 (7.1\%) (Fig. 6A,B), raising a possibility that the YFP/CFP-expressing OSNs might express some OR genes other than OR111-7 and OR103-1. Then we extended the double-labeling experiment for various OR genes located in the same cluster on chromosome 15. Individual OR members belonging to the OR111 subfamily (OR111-1, OR111-2, OR111-3, OR111-5, and OR111-10) were also expressed in small populations of GFP-immunoreactive OSNs (1.8-10.5\%). OR103-2 and/or OR103-5 belonging to the same subfamily as OR103-1 were expressed in a large population of GFP-immunoreactive OSNs (93.5\%). In contrast, we could not detect any GFP-immunoreactive OSNs that expressed OR102-1, OR107-1, or OR119-2 despite their localization in the same OR gene cluster as the OR111 and OR103 subfamilies (Fig. 6A,B). The majority of zebrafish OR genes are distributed in five large clusters on chromosomes 10,15, and 21 in the zebrafish genome (Alioto and Ngai, 2005; Niimura and Nei, 2005). When we examined expression of OR genes contained in other OR gene clusters, none of the tested OR genes was expressed in GFP-immunoreactive OSNs, except for OR106-11 (7.9\%) (Fig. 6A, B).

To confirm that the OR gene choice in GFP-immunoreactive OSNs in BAC-YC fish is not random but strictly regulated, we analyzed the expression rates of individual OR genes by estimating the numbers of OR probe-positive cells in an entire olfactory rosette of wild-type fish. Individual OR genes were expressed in disparate numbers of OSNs in the adult OE (Fig. 6C), and the profile of OR gene expression in GFP-immunoreactive OSNs in BAC-YC fish did not reflect the expression rate of individual OR genes in the $\mathrm{OE}$ at all. These data indicate that OR genes expressed in YFP/ CFP-expressing OSNs in BAC-YC fish are not randomly chosen according to the frequency of OR expression but are restricted within members of the OR111 and OR103 subfamilies to which the replaced ORs (OR111-7 and OR103-1) belong.

\section{A subpopulation of zebrafish OSNs express multiple OR genes}

In the analysis of OR gene expression in YFP/CFP-expressing OSNs in BAC-YC fish, summation of all of the percentages for lateral. Scale bar, $100 \mu \mathrm{m}$.
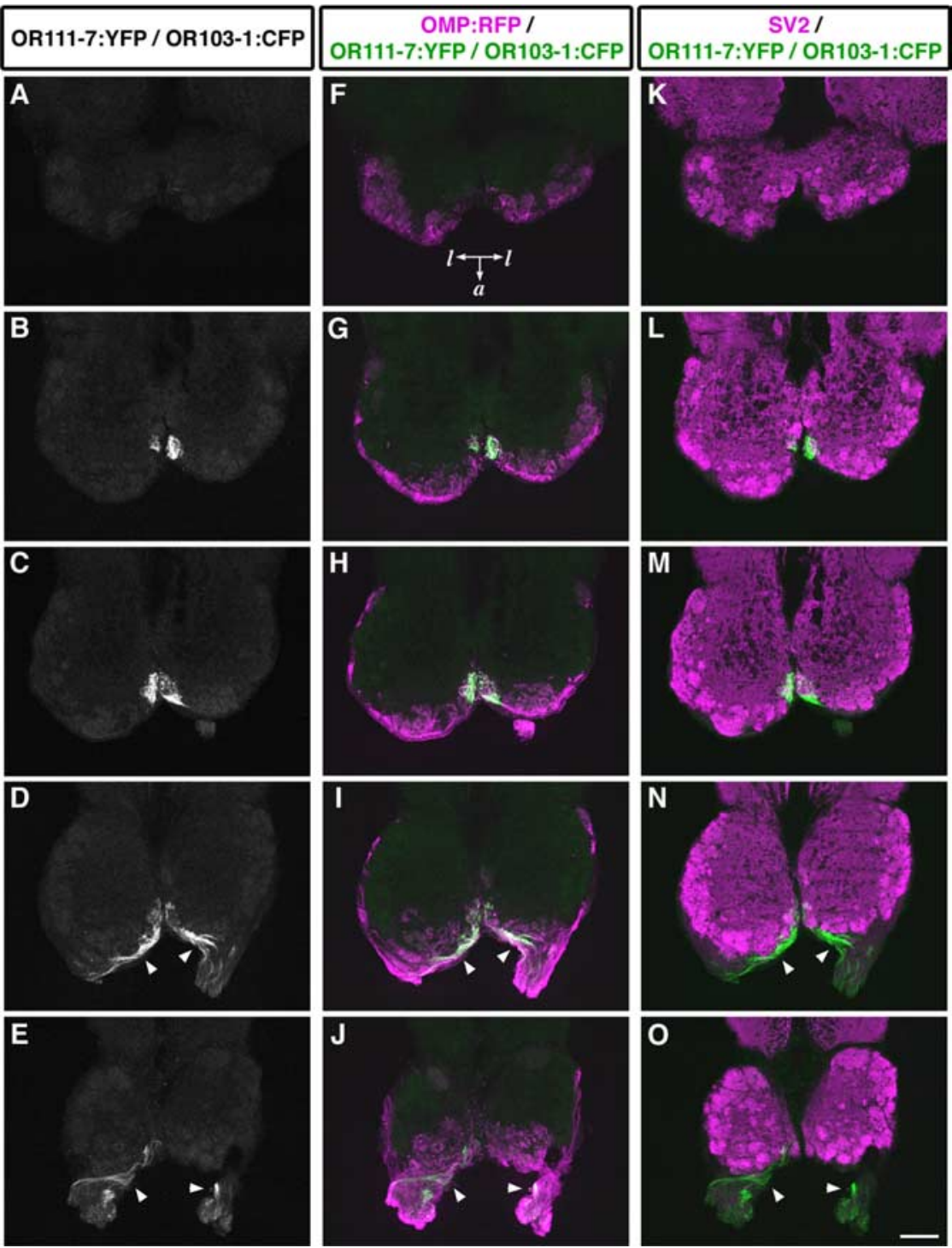

Figure 4. Targeting of YFP/CFP-expressing OSNs to specific glomeruli in the medial OB of adult $B A C-Y C$ fish. Horizontal sections through the OB of adult BAC-YC;OMP:RFP double-transgenic fish were triple labeled with anti-GFP, anti-RFP, and anti-SV2 antibodies. $\boldsymbol{A}-\boldsymbol{E}$, GFP immunoreactivities to YFP and (FP. $\boldsymbol{F}-\boldsymbol{J}$, Merged images of GFP (green) and RFP (magenta) immunoreac. (bottom). All photographs represent stacked images of optical sections. GFP-immunoreactive axons innervate a small population of RFP-labeled glomeruli in the medial $\mathrm{OB}$. Arrowheads in $\mathbf{D}, E, I, J$, and $\mathbf{N}, \mathbf{O}$ indicate $\mathrm{OSN}$ axons entering the $\mathrm{OB}$. a, Anterior; I,

OR-positive cells among GFP-immunoreactive OSNs reached $150 \%$, significantly exceeding $100 \%$ (Fig. $6 \mathrm{~B}$ ). This result suggests that there may be a substantial population of OSNs expressing multiple OR genes. To examine the possibility of multiple OR gene expression in single OSNs, we performed double in situ hybridization analysis for various combinations of OR members belonging to the OR111 and OR103 subfamilies (Fig. 7). In both wild-type and BAC-YC fish, all pairwise combinations of the OR111 subfamily members tested were expressed in nonoverlapping subsets of OSNs (Fig. 7A,B,E,F) (supplemental Table S2, available at www.jneurosci.org as supplemental material). These results are essentially consistent with the previous report showing that the OR111 subfamily members are expressed individually in different OSNs (Barth et al., 1997). To investigate whether OR members of the OR111 and OR103 subfamilies were coexpressed 

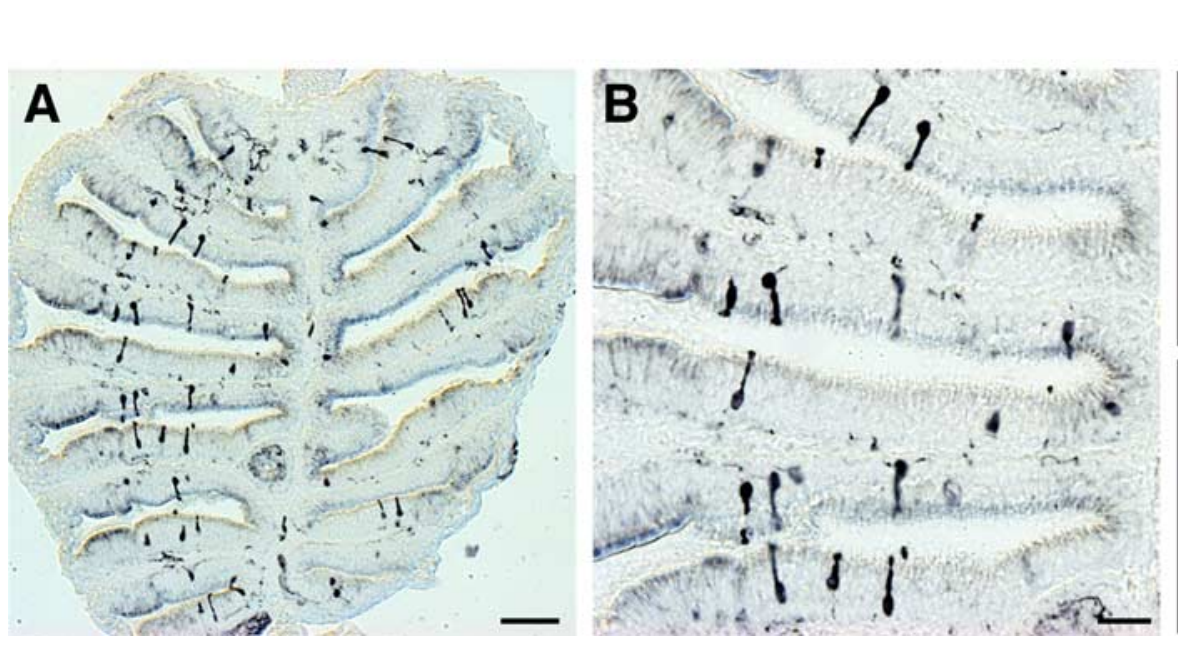

OR111-7:YFP /
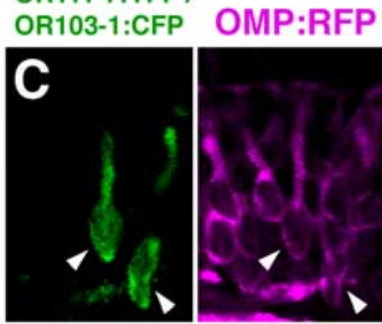

merge

Figure 5. Expression of YFP/CFP in ciliated OSNs of the OE from adult BAC-YC fish. A, Immunostaining of YFP and CFP with anti-GFP antibody was performed on horizontal sections of the OE from adult BAC-YC fish. Scale bar, $50 \mu \mathrm{m}$. B, A high-power view of $A$. GFP immunoreactivity is detected in OSNs with long dendrites. Scale bar, $20 \mu \mathrm{m}$. C, GFP immunoreactivity and RFP fluorescence on OE sections from adult BAC-YC;OMP:RFP double-transgenic fish. GFP immunoreactivity is detected in RFP-positive ciliated OSNs (arrowheads). Scale bar, $10 \mu \mathrm{m}$.

in the same OSN or not, double in situ hybridization was performed using mixed probes for the respective subfamilies. In wild-type fish, a cell population expressing the OR111 members is completely discrete from that expressing the OR103 members (Fig. 7C). In BAC-YC fish, however, coexpression of the OR111 and OR103 subfamily members was detected in 19 OSNs among 345 OR111-positive and 156 OR103-positive cells (Fig. 7G, arrowheads). We next examined coexpression of OR members within the OR103 subfamily. Surprisingly, OSNs labeled with the OR103-1 probe were always positive for a mixture of the OR103-2 and OR103-5 probes in both wild-type and BAC-YC fish (Fig. $7 \mathrm{D}, \mathrm{H})$. Because, in our hybridization experiments, crosshybridization did not occur even when cRNA probes with high sequence identities were used together (OR111-1 vs OR111-7, $81 \%$; OR111-7 vs OR111-3, 80\%), there would be much less possibility of cross-hybridization between OR103-1 and OR103-2/ OR103-5 (67\% identity). Colocalization of signals for OR103-1 and OR103-2/OR103-5, therefore, directly indicates that all OR103-1-positive OSNs simultaneously coexpress OR103-2 and/or OR103-5. Expression of multiple OR genes in a subpopulation of OSNs could provide an account for the excess in a sum of the percentages for OR-positive cells.

\section{Discussion}

In this study, we generated BAC transgenic zebrafish carrying an OR gene cluster in which the coding sequences of two OR-type receptors, OR111-7 and OR103-1, were replaced with YFP and CFP, respectively. We found that the choice of OR genes in YFP/ CFP-expressing OSNs was limited to OR members belonging to the OR111 and OR103 subfamilies and that the YFP/CFPexpressing OSNs expressing OR members of the OR111 and OR103 subfamilies converged their axons onto a topographically fixed glomerular cluster in the medial OB. These results demonstrate the hierarchical regulation of OR gene choice and the subsequent axonal convergence to defined glomeruli in the zebrafish olfactory system.

Hierarchical regulation of OR gene choice in zebrafish OSNs In BAC-YC (OR111-7:YFP/OR103-1:CFP) transgenic fish, OR genes expressed in $\mathrm{YFP} / \mathrm{CFP}$-expressing OSNs were not ran- domly distributed on various chromosomal loci but were mostly restricted within members of the OR111 and OR103 subfamilies (Fig. 6). Not only OR genes contained in BAC-YC transgene (OR111-5, OR111-10, and OR103-2/OR103-5) but also other OR genes located only in endogenous alleles (OR111-1, OR111-2, OR111-3, OR111-7, and OR103-1) were expressed in GFPimmunoreactive OSNs, in which either YFP or CFP gene within BAC-YC transgene was also activated for expression. These findings indicate that all OR members of the OR111 and OR103 subfamilies had a potential for expression in the YFP/CFPexpressing OSNs, regardless of their location either within BAC-YC transgene or on the endogenous allele. Thus, the genomic organization of OR subfamilies might provide a basis for regulation of OR expression in the zebrafish olfactory system.

Whereas OR choice in YFP/CFP-expressing OSNs was mostly restricted within the OR111 and OR103 subfamily members, OR106-11 mRNA was exceptionally detected in YFP/CFPexpressing OSNs among the tested OR genes belonging to different subfamilies. Although the chromosomal location of the OR106 subfamily (chromosome 10) is different from that of the OR111 and OR103 subfamilies (chromosome 15), the OR106 and OR111 subfamilies are classified into the same or closely related OR groups based on the phylogenic analysis (Alioto and Ngai, 2005; Niimura and Nei, 2005). Expression of the OR111 and OR106 subfamily members may be mediated by a common regulatory mechanism.

Individual OR111 subfamily members were expressed in nonoverlapping subsets of OSNs (Fig. 7), suggesting that OSNs, which are allowed to express OR111 subfamily members, would choose only one OR among the subfamily. One attractive mechanistic model to ensure the "one receptor-one neuron" rule emerged from several lines of evidence with transgenic mice (Serizawa et al., 2003; Lewcock and Reed, 2004; Shykind et al., 2004). Once a functional OR is expressed, the OR will prevent expression of additional OR genes through putative feedback signals. If the negative feedback regulation demonstrated in mice is also applicable to zebrafish, OSNs that initially express the YFP gene in BAC-YC fish would fail to produce a feedback signal and subsequently choose alternate ORs within the OR111 subfamily. 
This notion leads to a two-step model of the hierarchical regulation of OR gene choice in the zebrafish olfactory system (Fig. 8): (1) individual OSNs would initially choose one or a few OR subfamilies for expression, or, alternatively, the choice of OR subfamilies may be predetermined for individual OSNs according to their cell lineage or spatial localization in the OE; (2) the OSNs subsequently choose a single OR gene among the limited subfamily members.

\section{Similarities in OR gene choice mechanisms between zebrafish and mice}

Our hypothetical model of hierarchical OR gene choice in zebrafish might be similar to the mode of OR gene choice in mice within the meaning that individual OSNs choose a single OR gene among limited OR members. In mice, ORs are approximately classified into four groups according to their expression zones in the $\mathrm{OE}$ (Ressler et al., 1993; Vassar et al., 1993; Sullivan et al., 1996), although two recent papers reported that patterns of OR gene expression are arranged not in conventional zones but rather in continuous and overlapping domains (Iwema et al., 2004; Miyamichi et al., 2005). Switching of OR gene choice in OSNs expressing a mutant OR is permitted within the repertoire of OR genes in the same zone (Serizawa et al., 2003; Shykind et al., 2004). In a given zone, structurally related ORs belonging to same subfamilies are generally expressed, which may underlie the zonal difference in reception of odors with distinct qualities (Mori et al., 2000). Some zebrafish ORs also show concentric expression patterns within the OE (Weth et al., 1996). If zonal patterns of OR expression are related to the classification of OR subfamilies in zebrafish, it is likely that a common hierarchical mechanism of OR gene choice is used between zebrafish and mice.

A recent report demonstrated that an enhancer element, $H$ region, can mediate the stochastic, interchromosomal transactivation of a single OR gene locus in mouse OSNs (Lomvardas et al., 2006). The presence of a trans-acting enhancer element homologous to the mouse $H$ region has not been proved in zebrafish. However, a similar enhancer-mediated transcriptional mechanism may be used also in zebrafish in the process of a single OR gene choice among limited OR subfamily members.

\section{Expression of multiple ORs in single OSNs}

A double-labeling experiment for YFP/CFP and OR mRNA expression in BAC-YC fish yielded a curious but interesting result: a sum of the percentages for OR-positive cells among YFP/CFP-
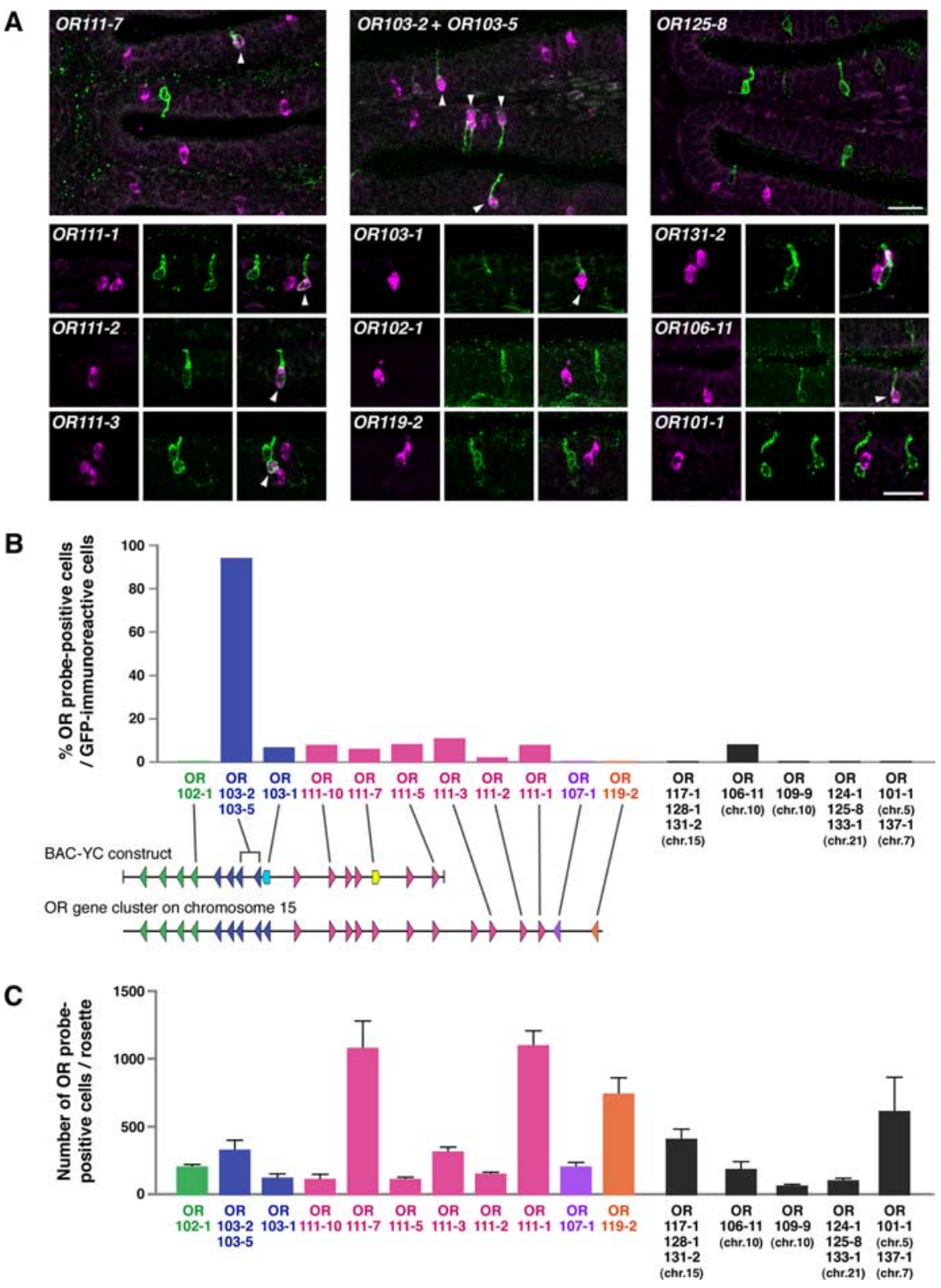

Figure 6. Expression of OR genes belonging to restricted subfamilies in YFP/CFP-expressing OSNs. A, OE sections from adult $B A C-Y C$ fish were hybridized with CRNA probes for various $O R$ genes (magenta) and subsequently immunostained with anti-GFP antibody (green). The arrowheads indicate GFP-immunoreactive OSNs that are labeled with the OR gene probe tested. Scale bars, $20 \mu \mathrm{m}$. $\boldsymbol{B}$, Percentages of the $\mathrm{OR}$ probe-positive cells among GFP-immunoreactive cells are shown in relation to an array of $O R$ genes in the cluster on chromosome 15. OR genes expressed in GFP-immunoreactive OSNs are mostly restricted within the OR111 and OR103 subfamilies. C, Numbers of OSNs labeled with individual OR probes in an entire olfactory rosette from adult wild-type zebrafish. To estimate the numbers of OSNs expressing individual OR genes in an entire olfactory rosette, in situ hybridization was performed on 0 E sections from adult wild-type fish. For each $O R$ probe, the total number of $O R$ probe-positive cells on every four slices from serial sections is quadruplicated and shown as the estimated number of $O R$ probe-positive cells per an olfactory rosette. Data represent means \pm SEM $(n=3-7)$. Individual OR genes are expressed in different numbers of OSNs in the wild-type 0 E with no relation to the OR choice in YFP/CFP-expressing OSNs in BAC-YC fish. Mixed OR probes were used as follows: OR103-2 and OR103-5; OR117-1, OR128-1, and OR131-2 on chromosome 15; OR124-1, OR125-8, and OR133-1 on chromosome 21; OR101-1 on chromosome 5; and 0R137-1 on chromosome 7.

expressing OSNs reached $150 \%$ (Fig. 6B). This finding prompted us to examine a possibility that a subpopulation of OSNs may express multiple OR genes. In BAC-YC fish, double in situ hybridization analysis demonstrated the coexpression of ORs in a small but significant population of OSNs: (1) OR103-1expressing OSNs were always positive for OR103-2 and/or OR103-5 (Fig. 7H); (2) coexpression of the OR111 and OR103 subfamily members was observed in a small population of OSNs 


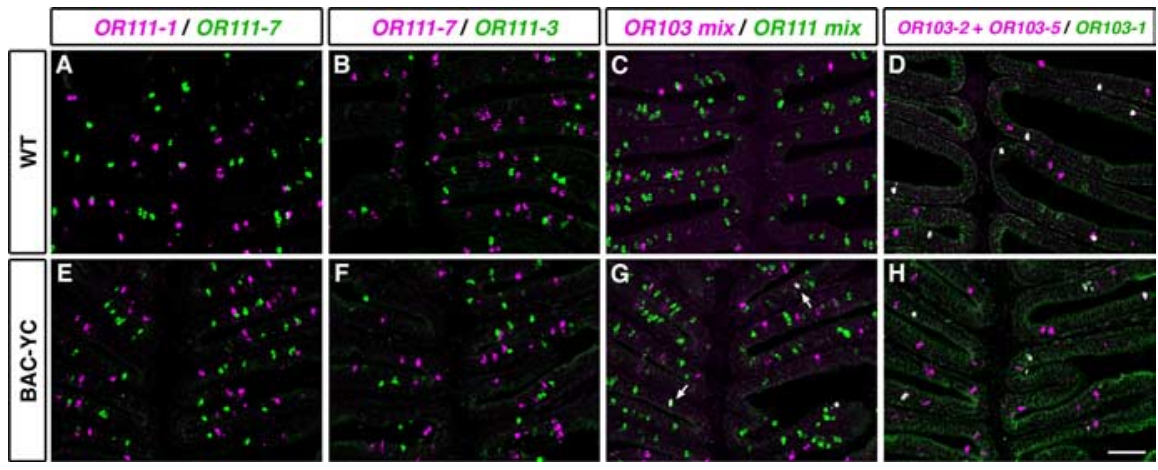

Figure 7. Coexpression of multiple OR receptors in single OSNs in adult wild-type (WT) and BAC-YC (OR111-7:YFP/OR103-1: (FP) fish. Double in situ hybridization was performed on OE sections from wild-type $(\boldsymbol{A}-\boldsymbol{D})$ and $B A C-Y C(\boldsymbol{E}-\boldsymbol{H})$ fish with various combinations of OR members belonging to the OR111 and OR103 subfamilies. $A, E$, Nonoverlapping expression of OR111-1 (magenta) and OR111-7 (green) in wild-type $(\boldsymbol{A})$ and BAC-YC $(\boldsymbol{E})$ fish. $\boldsymbol{B}, \boldsymbol{F}$, Nonoverlapping expression of OR111-7 (magenta) and OR111-3 (green) in wild-type $(\boldsymbol{B})$ and BAC-YC $(\boldsymbol{F})$ fish. $\boldsymbol{C}, \boldsymbol{G}$, Expression of ORs belonging to the OR103 (magenta) and OR111 (green) subfamilies. Mixed probes for the OR103 subfamily contains probes for OR103-1, OR103-2, and OR103-5. Mixed probes for the OR111 subfamily contains probes for OR111-1, OR111-2, OR111-3, OR111-5, OR111-7, and OR111-10. No overlapping signal is observed in wild-type fish $(\boldsymbol{C})$, whereas overlapping signals are detected at low frequency in $\mathrm{BAC}-\mathrm{YC}$ fish (arrows in $\mathbf{G})$. $\boldsymbol{D}, \boldsymbol{H}$ Coexpression of OR103-1 (green) with OR103-2 and/or OR103-5 (magenta) in both wild-type (D) and BAC-YC (H) fish. All OSNs labeled with the OR103-1 probe are also positive for a mixture of the OR103-2 and OR103-5 probes (shown in white). All photographs represent stacked images of optical sections. Scale bar, $50 \mu \mathrm{m}$. Wild-type zebrafish

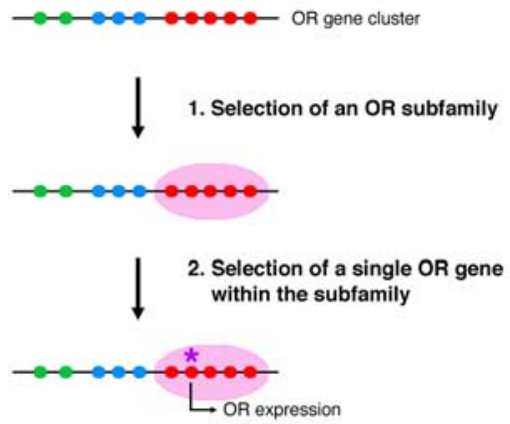

B

OR-deleted transgenic zebrafish

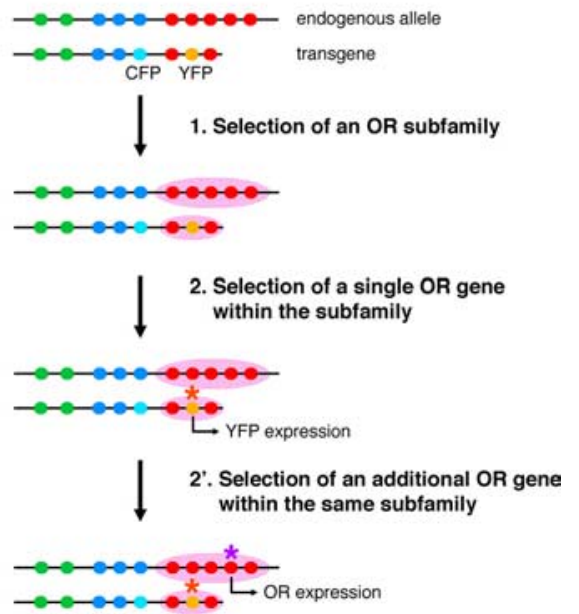

Figure 8. A model of hierarchical regulation of zebrafish OR gene choice. $A, A$ single OR gene for expression is determined by the two-step selection in OSNs. For individual OSNs, the OR gene choice is initially confined to one or a few OR subfamilies (pink circles). The OSNs subsequently choose a single OR gene (a purple asterisk) among the limited subfamily members. $\boldsymbol{B}$, The 0 R gene choice in transgenic zebrafish in which OR-coding sequences are replaced with reporter genes. After the selection of an OR subfamily (pink circles), if OSNs choose a reporter gene (an orange asterisk) and fail to express a functional OR, the OSNs choose an additional OR gene (a purple asterisk) among the limited subfamily members. OR genes belonging to individual $O R$ subfamilies are represented by small circles of the same colors.

(Fig. 7G). These results indicate that the one receptor-one neuron rule is not always the case for a subpopulation of OSNs in the BAC-YC transgenic zebrafish. Because the coding sequence of OR103-1 is replaced with CFP in the BAC-YC transgene, CFPpositive OSNs should simultaneously express OR103-2 and/or OR103-5. This notion, together with the dominant number of CFP-expressing cells over YFP-expressing cells, may explain why a large population (93.5\%) of GFP-immunoreactive OSNs expressed OR103-2 and/or OR103-5 in BAC-YC fish.

Intriguingly, also in wild-type fish, OR103-1-positive OSNs simultaneously expressed OR103-2 and/or OR103-5 (Fig. 7D).
This finding clearly demonstrates that the one receptor-one neuron rule is not necessarily applicable to zebrafish OSNs and that the "multiple receptors-one neuron" rule is true at least for the OR103 subfamily members. Coexpression of functional chemosensory receptors in single neurons has been also reported in Drosophila and Caenorhabditis elegans (Troemel et al., 1995; Goldman et al., 2005). For example, the AWC neuron in C. elegans expresses multiple ORs, responds to various odorants such as aldehydes, ketones, alcohols, and thiazoles without discrimination, and mediates attractive behavior to all of these odorants (Bargmann et al., 1993; Troemel et al., 1995). By analogy, it may be unnecessary for zebrafish to discriminate odorant molecules received by the individual OR103 subfamily members. These OSNs expressing multiple OR103 members thus may integrate odor information at the most peripheral level, leading to a particular behavioral or hormonal response.

\section{Glomerular targeting of OSN axons in zebrafish}

Previous studies using molecular biological and genetic techniques demonstrated that, in Drosophila and mice, OSNs expressing a given OR project their axons onto one or a few topographically fixed glomeruli in the antennal lobe or the $\mathrm{OB}$ (Ressler et al., 1994; Vassar et al., 1994; Mombaerts et al., 1996; Gao et al., 2000; Vosshall et al., 2000). This principle of axonal convergence to target glomeruli underlies the development of odor maps in the brain (Mori et al., 1999). In zebrafish, the existence of an odor map was demonstrated by neural activity imaging of OSN axon terminals and $\mathrm{OB}$ projection neurons (Friedrich and Korsching, 1997, 1998; Li et al., 2005). Different classes of odor molecules (e.g., amino acids, nucleotides, bile acids, steroids, and prostaglandins) elicit distinct patterns of glomerular activity in the zebrafish OB. However, no direct evidence has been reported, to date, for axonal convergence of OSNs expressing the same type of OR genes onto specific glomeruli in zebrafish.

In this study, we analyzed in detail the relationship between OR gene choice and glomerular innervation of YFP/CFPexpressing OSNs in BAC-YC fish. The YFP/CFP-expressing OSNs expressed limited members of OR genes belonging mostly to the OR111 and OR103 subfamilies and projected their axons to a specific cluster of glomeruli in the medial portion of the OB. Such OR gene choice profiles and patterns of OSN axon projection were indistinguishable among different individuals. These findings indicate the axonal convergence of OSNs expressing OR genes belonging to particular subfamilies onto a topographically fixed glomerular cluster in the OB. Thus, the principle of "axonal 
convergence of like OSNs" seems to be also applicable to zebrafish, although the "one OR-one glomerulus" relationship remains to be elucidated.

In the fish olfactory system, two major types of OSNs, ciliated and microvillous OSNs, are contained in different layers of the OE. Previous studies have proposed that ciliated OSNs project their axons to mainly medial regions of the $\mathrm{OB}$ and mediate avoidance behaviors, whereas microvillous OSNs target lateral regions of the $\mathrm{OB}$ and mediate feeding behaviors (Hamdani et al., 2001; Hamdani and Døving, 2002; Hansen et al., 2003; Weltzien et al., 2003; Sato et al., 2005). An anteromedial portion of the zebrafish $\mathrm{OB}$ is activated by bile acids (Friedrich and Korsching, 1998), suggesting that a cluster of glomeruli targeted by YFP/ CFP-expressing OSNs in BAC-YC fish would also respond to bile acids. Biological function of bile acids is not clear in zebrafish, but, in sea lampreys, it has been reported that bile acids function as migratory attractants (Li et al., 2002; Sorensen et al., 2005). Additional experiments will clarify whether ORs belonging to the OR111 and OR103 subfamilies are actually the receptors specific for bile acids or not.

\section{References}

Alioto TS, Ngai J (2005) The odorant receptor repertoire of teleost fish. BMC Genomics 6:173.

Baier H, Korsching S (1994) Olfactory glomeruli in the zebrafish form an invariant pattern and are identifiable across animals. J Neurosci 14:219-230.

Bargmann CI, Hartwieg E, Horvitz HR (1993) Odorant-selective genes and neurons mediate olfaction in C. elegans. Cell 74:515-527.

Barth AL, Justice NJ, Ngai J (1996) Asynchronous onset of odorant receptor expression in the developing zebrafish olfactory system. Neuron 16:23-34.

Barth AL, Dugas JC, Ngai J (1997) Noncoordinate expression of odorant receptor genes tightly linked in the zebrafish genome. Neuron 19:359-369.

Cao Y, Oh BC, Stryer L (1998) Cloning and localization of two multigene receptor families in goldfish olfactory epithelium. Proc Natl Acad Sci USA 95:11987-11992.

Chess A, Simon I, Cedar H, Axel R (1994) Allelic inactivation regulates olfactory receptor gene expression. Cell 78:823-834.

Dugas JC, Ngai J (2001) Analysis and characterization of an odorant receptor gene cluster in the zebrafish genome. Genomics 71:53-65.

Feinstein P, Mombaerts P (2004) A contextual model for axonal sorting into glomeruli in the mouse olfactory system. Cell 117:817-831.

Friedrich RW, Korsching SI (1997) Combinatorial and chemotopic odorant coding in the zebrafish olfactory bulb visualized by optical imaging. Neuron 18:737-752.

Friedrich RW, Korsching SI (1998) Chemotopic, combinatorial, and noncombinatorial odorant representations in the olfactory bulb revealed using a voltage-sensitive axon tracer. J Neurosci 18:9977-9988.

Gao Q, Yuan B, Chess A (2000) Convergent projections of Drosophila olfactory neurons to specific glomeruli in the antennal lobe. Nat Neurosci 3:780-785

Goldman AL, Van der Goes van Naters W, Lessing D, Warr CG, Carlson JR (2005) Coexpression of two functional odor receptors in one neuron. Neuron 45:661-666.

Hamdani EH, Døving KB (2002) The alarm reaction in crucian carp is mediated by olfactory neurons with long dendrites. Chem Senses 27:395-398.

Hamdani EH, Alexander G, Døving KB (2001) Projection of sensory neurons with microvilli to the lateral olfactory tract indicates their participation in feeding behaviour in crucian carp. Chem Senses 26:1139-1144.

Hansen A, Rolen SH, Anderson K, Morita Y, Caprio J, Finger TE (2003) Correlation between olfactory receptor cell type and function in the channel catfish. J Neurosci 23:9328-9339.

Hansen A, Anderson KT, Finger TE (2004) Differential distribution of olfactory receptor neurons in goldfish: structural and molecular correlates. J Comp Neurol 477:347-359.
Inaki K, Nishimura S, Nakashiba T, Itohara S, Yoshihara Y (2004) Laminar organization of the developing lateral olfactory tract revealed by differential expression of cell recognition molecules. J Comp Neurol 479:243-256.

Iwema CL, Fang H, Kurtz DB, Youngentob SL, Schwob JE (2004) Odorant receptor expression patterns are restored in lesion-recovered rat olfactory epithelium. J Neurosci 24:356-369.

Kimmel CB, Ballard WW, Kimmel SR, Ullmann B, Schilling TF (1995) Stages of embryonic development of the zebrafish. Dev Dyn 203:253-310.

Lalioti MD, Heath JK (2001) A new method for generating point mutations in bacterial artificial chromosomes by homologous recombination in Escherichia coli. Nucleic Acids Res 29:e14.

Lewcock JW, Reed RR (2004) A feedback mechanism regulates monoallelic odorant receptor expression. Proc Natl Acad Sci USA 101:1069-1074.

Li J, Mack JA, Souren M, Yaksi E, Higashijima S, Mione M, Fetcho JR, Friedrich RW (2005) Early development of functional spatial maps in the zebrafish olfactory bulb. J Neurosci 25:5784-5795.

Li W, Scott AP, Siefkes MJ, Yan H, Liu Q, Yun SS, Gage DA (2002) Bile Acid secreted by male sea lamprey that acts as a sex pheromone. Science 296:138-141.

Lomvardas S, Barnea G, Pisapia DJ, Mendelsohn M, Kirkland J, Axel R (2006) Interchromosomal interactions and olfactory receptor choice. Cell 126:403-413.

Macdonald R (1999) Zebrafish immunohistochemistry. In: Molecular methods in developmental biology: Xenopus and zebrafish (Guille M, ed), pp 77-88. Totowa, NJ: Humana.

Malnic B, Hirono J, Sato T, Buck LB (1999) Combinatorial receptor codes for odors. Cell 96:713-723.

Miyamichi K, Serizawa S, Kimura HM, Sakano H (2005) Continuous and overlapping expression domains of odorant receptor genes in the olfactory epithelium determine the dorsal/ventral positioning of glomeruli in the olfactory bulb. J Neurosci 25:3586-3592.

Miyasaka N, Sato Y, Yeo SY, Hutson LD, Chien CB, Okamoto H, Yoshihara Y (2005) Robo2 is required for establishment of a precise glomerular map in the zebrafish olfactory system. Development 132:1283-1293.

Mombaerts P, Wang F, Dulac C, Chao SK, Nemes A, Mendelsohn M, Edmondson J, Axel R (1996) Visualizing an olfactory sensory map. Cell 87:675-686.

Mori K, Nagao H, Yoshihara Y (1999) The olfactory bulb: coding and processing of odor molecule information. Science 286:711-715.

Mori K, von Campenhausen H, Yoshihara Y (2000) Zonal organization of the mammalian main and accessory olfactory systems. Philos Trans R Soc Lond B Biol Sci 355:1801-1812.

Morita Y, Finger TE (1998) Differential projections of ciliated and microvillous olfactory receptor cells in the catfish, Ictalurus punctatus. J Comp Neurol 398:539-550.

Ngai J, Chess A, Dowling MM, Necles N, Macagno ER, Axel R (1993) Coding of olfactory information: topography of odorant receptor expression in the catfish olfactory epithelium. Cell 72:667-680.

Niimura Y, Nei M (2005) Evolutionary dynamics of olfactory receptor genes in fishes and tetrapods. Proc Natl Acad Sci USA 102:6039-6044.

Ressler KJ, Sullivan SL, Buck LB (1993) A zonal organization of odorant receptor gene expression in the olfactory epithelium. Cell 73:597-609.

Ressler KJ, Sullivan SL, Buck LB (1994) Information coding in the olfactory system: evidence for a stereotyped and highly organized epitope map in the olfactory bulb. Cell 79:1245-1255.

Sato Y, Miyasaka N, Yoshihara Y (2005) Mutually exclusive glomerular innervation by two distinct types of olfactory sensory neurons revealed in transgenic zebrafish. J Neurosci 25:4889-4897.

Serizawa S, Miyamichi K, Nakatani H, Suzuki M, Saito M, Yoshihara Y, Sakano H (2003) Negative feedback regulation ensures the one receptor-one olfactory neuron rule in mouse. Science 302:2088-2094.

Shykind BM, Rohani SC, O’Donnell S, Nemes A, Mendelsohn M, Sun Y, Axel R, Barnea G (2004) Gene switching and the stability of odorant receptor gene choice. Cell 117:801-815.

Sorensen PW, Fine JM, Dvornikovs V, Jeffrey CS, Shao F, Wang J, Vrieze LA, Anderson KR, Hoye TR (2005) Mixture of new sulfated steroids functions as a migratory pheromone in the sea lamprey. Nat Chem Biol 1:324-328.

Speca DJ, Lin DM, Sorensen PW, Isacoff EY, Ngai J, Dittman AH (1999) Functional identification of a goldfish odorant receptor. Neuron 23:487-498. 
Sullivan SL, Adamson MC, Ressler KJ, Kozak CA, Buck LB (1996) The chromosomal distribution of mouse odorant receptor genes. Proc Natl Acad Sci USA 93:884-888.

Troemel ER, Chou JH, Dwyer ND, Colbert HA, Bargmann CI (1995) Divergent seven transmembrane receptors are candidate chemosensory receptors in C. elegans. Cell 83:207-218.

Vassar R, Ngai J, Axel R (1993) Spatial segregation of odorant receptor expression in the mammalian olfactory epithelium. Cell 74:309-318.

Vassar R, Chao SK, Sitcheran R, Nuñez JM, Vosshall LB, Axel R (1994) Topographic organization of sensory projections to the olfactory bulb. Cell 79:981-991.

Vosshall LB, Wong AM, Axel R (2000) An olfactory sensory map in the fly brain. Cell 102:147-159.
Wang F, Nemes A, Mendelsohn M, Axel R (1998) Odorant receptors govern the formation of a precise topographic map. Cell 93:47-60.

Weltzien FA, Höglund E, Hamdani EH, Døving KB (2003) Does the lateral bundle of the medial olfactory tract mediate reproductive behavior in male crucian carp? Chem Senses 28:293-300.

Westerfield M (1995) The zebrafish book. Eugene, OR: University of Oregon.

Weth F, Nadler W, Korsching S (1996) Nested expression domains for odorant receptors in zebrafish olfactory epithelium. Proc Natl Acad Sci USA 93:13321-13326.

Yang XW, Model P, Heintz N (1997) Homologous recombination based modification in Escherichia coli and germline transmission in transgenic mice of a bacterial artificial chromosome. Nat Biotechnol 15:859-865. 